\title{
Practical Phototherapy of Psoriasis
}

\section{Zusammenfassung}

Die Photo- und Photochemotherapie besitzen bei der Behandlung der Psoriasis einen sehr hohen Stellenwert. Allerdings sollte eine Phototherapie möglichst immer mit topischen oder, fakultativ, auch mit systemischen Medikamenten kombiniert werden; bei einer Photochemotherapie sind Kombinationsbehandlungen meist sinnvoll, aber nicht obligat. Zur UV-B-Phototherapie werden bevorzugt 311-nm-Schmalspektrum-Strahler eingesetzt. Eine Kombination mit Solebädern scheint die Wirkung zu verstärken. Es ist empfehlenswert, als erste therapeutische UV-Dosis $70 \%$ der vorher ermittelten MED zu verabfolgen. Alternativ kann auch entsprechend dem Photohauttyp nach einem standardisierten Schema vorgegangen werden. Der Stellenwert der Excimer-Laser-Therapie ist noch nicht ermittelt. Sie eignet sich für kleinere umschriebene Areale. Weitere Studien zur Wirksamkeit, Dauer der Rezidivfreiheit und zu Nebenwirkungen sind erforderlich. Zur Photochemotherapie stehen grundsätzlich neben der systemischen oralen auch topische Anwendungen des Photosensibilisators durch Vollbäder, Teilbäder und Cremes zur Verfügung. Bei allen Therapieformen besteht ein akutes Risiko für überschießende phototoxische Reaktionen und ein potenzielles Langzeit-Karzinomrisiko. Eine Kontraindikation ist eine Kombinationsbehandlung mit Cyclosporin A. Besondere Vorsicht ist geboten bei Vorbehandlungen mit Arsen, Röntgenstrahlen bei gleichzeitiger Einnahme photosensibilisierender Medikamente und bei ausgeprägter Leberschädigung. Eine Kombination der Photo(chemo)therapie mit Methotrexat ist möglich; zur Kombination mit systemischen Fumaraten existieren keine Erfahrungsberichte. Wünschenswert ist die Ermittlung der UV-A-Erstdosis durch die Bestimmung der MPD, wobei nach systemischer Sensibilisierung 50 bis $70 \%$ der MPD und nach topischer Sensibilisierung $30 \%$ der MPD als Erstdosis verwendet werden. Alternativ

\section{Abstract}

Photo- and photochemotherapy are first-line treatments for psoriasis. Phototherapy, however, should always be combined with topical or, optionally, with systemic drugs. In photochemotherapy combinations are useful, but not mandatory. For UVB-phototherapy preferably $311 \mathrm{~nm}$ narrow-band sources are used. The combination with brine-baths seems to inhance efficacy. It is suggested to use $70 \%$ of the MED as starting dose. A standardized regimen according to the photo-skintype is an appropriate alternative. The therapeutic use of excimer lasers has not been determined yet. It may be useful to treat localized recalcitrant lesions. Further studies on efficacy, duration of remission and side effects are indicated. Besides systemic oral photochemotherapy topical applications of sensitizer by whole body baths, palmoplantar soaks or application of creams are possible. All these applications confer an acute risk of overshooting phototoxic reactions and a potential long-term risk of skin carcinoma. Combination therapy with cyclosporine A is contra-indicated. Special caution must be exerted in case of pre-treatment with arsenic, X-ray or simultaneous medication with photosensitizer as well as in liver dysfunction. Combining photochemotherapy with methotrexate is possible; experience in using systemic furmarates in combination with photo(chemo)therapy is lacking. It is useful to determine the MPD before starting photochemotherapy. Using a systemic photosensitizer $50 \%$ to $70 \%$ of the MPD and using a topical photosensitizer $30 \%$ of the MPD should be used as initial dose. Again, a standardized regimen according to the photo-skintype can be employed instead. General guidelines for photo(chemo)therapy include strict adherence to indications and contraindications, close guidance of the patients during therapy, exact documentation of the UV-doses in physical units, regular follow ups, and complete protection of the eyes during the the- 
kann auch ein standardisiertes Verfahren entsprechend dem Photohauttyp erfolgen. Allgemeine Richtlinien zur Durchführung einer Photo(chemo)therapie umfassen die Beachtung von Indikationen und Kontraindikationen, eine straffe Patientenführung, eine exakte Dokumentation, auch der applizierten UV-Dosen in strahlungsphysikalischen Einheiten, eine regelmäßige ärztliche Überwachung einschließlich Nachuntersuchungen und einen vollständigen Augenschutz während der therapeutischen Bestrahlungen. Als absolute Kontraindikation gelten Gendefekte mit einer erhöhten Lichtempfindlichkeit oder einem erhöhten Hautkrebsrisiko, wie z.B. Xeroderma pigmentosum, Cockayne-Syndrom und Bloom-Syndrom. Strenge Indikationsstellung, engmaschige Kontrollen und modifizierte Behandlungsschemata erfordern Patienten mit Krampfleiden, mit der unvermeidbaren Einnahme von photosensibilisierenden Medikamenten, mit malignen Hauttumoren in der Vorgeschichte, mit erhöhter Lichtempfindlichkeit und Patienten mit atypischen (dysplastischen) Nävuszellnävi. rapeutic irradiations. Absolute contra-indications are gene defects associated with increased photosensitivity or an increased risk of skin cancer as, for example, xeroderma pigmentosum, Cockayne's-syndrome, and Bloom's-syndrome. Patients with epilepsy, with inevitable photosensitizing medication, a history of malignant skin tumors, increased general photosensitivity, and with atypical nevi need special precautions including modified therapeutic regimens.

\section{Einleitung}

Photo- und Photochemotherapie besitzen bei der Behandlung der Psoriasis einen sehr hohen Stellenwert. Für die reine Phototherapie hat sich die Anwendung der Schmalspektrum UVB-311-nm-Therapie [1] durchgesetzt. Die Bade-PUVA-Behandlung wäre in der Lage, die systemische Photochemotherapie weitgehend zu ersetzen, dem steht jedoch die Ablehnung der Kostenübernahme durch die Kassen entgegen. In den USA ist seit etwa 10 Jahren ein deutlicher Rückgang der UV-Photo(chemo)therapie bei der Psoriasis zu verzeichnen [2]. Gründe hierfür bilden der hohe Aufwand des Therapeuten, die geringe Erstattung durch die Kassen, eine notwendige Zuzahlung durch die Patienten sowie die zunehmende Entwicklung und schrittweise Zulassung hochwirksamer systemischer Medikamente.

In der vorliegenden Übersichtsarbeit werden der gegenwärtige Stand der Photo(chemo)therapie dargestellt und strukturelle Voraussetzungen für die Behandlung, Therapieschemata, Indikationen und Kontraindikationen vorgeschlagen und diskutiert. Die verfahrenstechnischen Ausführungen basieren auf den jüngst als Leitlinie publizierten „Empfehlungen zur Phototherapie und Photochemotherapie“ [3].

\section{Phototherapieverfahren und Kombinationen}

Als Möglichkeiten der UV-Therapie stehen systemische PUVA, Bade-PUVA, Breitband-UV-B-Phototherapie, Schmalspektrum311-nm-Phototherapie und die Photosole-Behandlung mit Breitband- oder 311-nm-UV-B-Phototherapie, zur Verfügung. Um die Wirksamkeit der Phototherapie zu verbessern und damit die UVBelastung des Patienten zu minimieren, empfehlen sich Kombinationsbehandlungen [4]. Möglich sind einerseits Kombinationen mit Externa wie Cignolin, Vitamin D und Vitamin-D-Analoga, Tazaroten und Steroiden, und andererseits mit systemischen Medikamenten. Letztere werden jedoch in ihrem Risikoprofil unterschiedlich bewertet. Eine Kombination mit Retinoiden (Acetritin) ist empfehlenswert, wenn die Kontraindikationen des Medika- ments beachtet werden. Die Anwendung von systemischen Kortikosteroiden zusätzlich zur Photo(chemo)therapie ist zwar möglich, die Indikationen hierzu sollten jedoch streng gestellt werden. Eine Mehrfachkombination von Photo(chemo)therapie mit Retinoiden und Steroiden ist zum Beispiel bei Patienten mit systemischer pustulierender Psoriasis sinnvoll. Die Kombination mit Fumaraten ist prinzipiell möglich, es gibt derzeit jedoch keine publizierten Erfahrungsberichte und das Langzeitrisiko ist unbekannt. Da Fumarate als milde Immunsuppressiva wirken, könnte das Karzinomrisiko durch eine begleitende Photo(chemo)therapie erhöht werden. Eine Kombination mit Methotrexat ist in den USA üblich und die Langzeitbeobachtung, insbesondere von PUVA-Patienten, konnte keine weitere Erhöhung des Karzinomrisikos durch Methotrexat darstellen. Eine Kombination von Photochemotherapie und Cyclosporin A ist nicht indiziert, da sich hierbei die Risiken für die Karzinomentwicklung an der Haut von Photochemotherapie und Cyclosporin synergistisch erhöhen. So berichten Marcil und Stern [5] über eine Gruppe von 28 Patienten, die in eine Langzeit-PUVA-Beobachtung eingeschlossen waren und die gleichzeitig während dieser Zeit mit Cyclosporin behandelt wurden. Vor der Cyclosporin-Anwendung fand sich bei sechs aus 28 (21\%) Patienten ein spinozelluläres Karzinom (20 Tumoren), nach Cyclosporin-Anwendung zeigten 13 von 28 (46\%) insgesamt 169 Karzinome. Die Gesamtinzidenz für das spinozelluläre Karzinom stieg nach der Anwendung von Cyclosporin A um das 6,9fache. In einer jüngst veröffentlichten Studie von Paul et al. [6] wurden 1252 Psoriasispatienten, die mit Cyclosporin behandelt wurden, über fünf Jahre beobachtet. Insgesamt hatten sie ein um das Sechsfache erhöhtes Risiko, maligne epitheliale Hauttumoren zu entwickeln. Zusätzliche Risikofaktoren waren eine Behandlung von Cyclosporin über einen längeren Zeitraum als zwei Jahre sowie die Kombination von Cyclosporin und PUVA.

\section{1-nm-Phototherapie}

Die 311-nm-Phototherapie besitzt im Vergleich zur herkömmlichen Breitband-UV-Phototherapie eine erhöhte Wirksamkeit und größere therapeutische Breite [7,8]. Weitere Studien ver- 
suchten die optimale Therapiefrequenz und die günstigste Dosierung herauszuarbeiten. Dawe et al. [9] führten an 21 Psoriasispatienten einen Halbseitenversuch durch, bei dem sie eine dreimal wöchentliche gegen eine fünfmal wöchentliche Applikation verglichen. Bis zur weitgehenden Erscheinungsfreiheit waren bei dreimal wöchentlicher Anwendung insgesamt 40 Tage mit $17 \mathrm{Be}-$ handlungen notwendig, und es wurde eine Gesamtdosis von 64 minimalen Erythemdosen appliziert. Bei fünfmal wöchentlicher Anwendung wurde Erscheinungsfreiheit nach 35 Tagen mit insgesamt 23 Behandlungen und einer Gesamtdosis von 94 MED's erreicht. Die Autoren empfehlen als Fazit aus dieser Studie eine Behandlung dreimal pro Woche. Zu einem ähnlichen Ergebnis kamen Cameron et al [10] in einem Gruppenvergleich an 130 Psoriasispatienten bei der Gegenüberstellung zwischen zwei und drei Bestrahlungen pro Woche. Die dreimal wöchentliche Anwendung war deutlich überlegen. Die größere therapeutische Breite der 311-nm-Phototherapie wird durch eine bereits hohe Effektivität von Suberythemdosen gewährleistet. Hofer et al [8] verglichen an 11 Psoriasispatienten in einem Halbseitenversuch die Anwendung einer Einzeldosis von 70\% der MED mit 50\% der MED. Die Therapieergebnisse unterschieden sich nicht signifikant, die Gesamtdosis war jedoch durch die Reduktion der Einzeldosen deutlich vermindert. Die Autoren empfehlen daher die Halbierung der bisher üblichen Anfangsdosen in den vorgeschlagenen Therapieschemata. In einem ähnlichen Halbseitenansatz wurden Suberythemdosen von 311-nm versus Breitband-UV-B verglichen. Eine signifikante Therapieverbesserung konnte nur durch die Schmalspektrumbehandlung erreicht werden. 9 von 11 Patienten zeigten eine komplette Remission. Diese konnte nur bei einem von 11 Patienten durch die Breitbandbehandlung erzielt werden [11].

In kontrollierten Studien zeigt sich die 311-nm-Phototherapie einer PUVA-Behandlung annähernd ebenbürtig. So zeigten Markham et al. [12] in einem Gruppenvergleich an 54 Psoriasispatienten, dass zwischen der dreimal wöchentlichen Anwendung von Schmalspektrum-UV und der zweimal wöchentlichen Anwendung von PUVA oral kein signifikanter Unterschied bestand. Zu ähnlichen Ergebnissen gelangten auch andere Autoren [13], wobei allerdings bei schwerer Psoriasis die PUVA-Therapie einen Therapievorteil zeigte.

\section{Photosoletherapie}

Sie ist die Umsetzung der Balneo-Klimatherapie, wie sie z.B. in den Heilbädern der Nord- und Ostsee, in Kureinrichtungen mit natürlicher Starksole und am Toten Meer durchgeführt wird. Meist wird im Anschluss an ein Vollbad in einer Salzlösung mit UV-B bestrahlt. Auch eine synchrone Bestrahlung während des Badens ist möglich. Bei der Psoriasis werden konzentrierte Lösungen bis zu $15 \%$ verwendet. Es wird angenommen, dass durch die Einwirkung der Salzlösung die optischen Eigenschaften der Hornschicht verändert und insbesondere proinflammatorische Mediatoren eluiert werden.

\section{Praktische Durchfüihrung der Phototherapie}

Vor Beginn der Phototherapie ist es empfehlenswert, die individuelle Erythemempfindlichkeit (MED) zu ermitteln. Hierzu geeignete Testdosen werden in der Tab. 1 vorgeschlagen. Alternativ kann auch entsprechend dem Photohauttyp nach einem standardisierten Schema vorgegangen werden (Tab. 2). Etwa 70\% der durch Phototestung ermittelten MED entsprechen der ersten therapeutischen UV-Dosis. Da der Erythemgipfel bereits 12 bis 24 Stunden nach der vorangegangenen Bestrahlung auftritt, kann die Dosis prinzipiell bei jeder Behandlungssitzung gesteigert werden. Die UV-Behandlung sollte drei- bis fünfmal wöchentlich durchgeführt werden. Die Steigerungsrate hängt von der Wirkung der vorausgegangenen Bestrahlung ab und kann zwischen 10 und $30 \%$ variieren. Die Tab. 3 zeigt ein praxisbewährtes Behandlungsschema.

Tab. 1 Dosisempfehlungen für die UV-B-Lichttreppen $\left(\mathrm{m} / / \mathrm{cm}^{2}\right)$

\begin{tabular}{lrrrrrr}
\hline UV-B-Breitband & 20 & 40 & 60 & 80 & 100 & 120 \\
\hline UV-B-311-nm & 200 & 400 & 600 & 800 & 1000 & 1200 \\
\hline
\end{tabular}

Tab. 2 Empfehlungen für die Anfangsdosis bei UV-B-Phototherapie

\begin{tabular}{lll}
\hline Photohauttyp & $\begin{array}{l}\text { UV-B-Breitband } \\
\mathbf{m J} / \mathbf{c m}^{\mathbf{2}}\end{array}$ & $\begin{array}{l}\text { UV-B-311-nm } \\
\mathbf{m J} / \mathbf{c m}^{\mathbf{2}}\end{array}$ \\
\hline I & & \\
II & 20 & 200 \\
III & 30 & 300 \\
\hline IV & 50 & 500 \\
\hline
\end{tabular}

Tab. 3 Dosisschema für die UV-B-Phototherapie (UV-Breitband und UV-B-311-nm)

\begin{tabular}{|c|c|c|c|}
\hline Schritt 1 & $\begin{array}{l}\text { Bestimmung der } \\
\text { MED }\end{array}$ & $\begin{array}{l}\text { Ablesung nach } \\
24 \text { Std. }\end{array}$ & \\
\hline Schritt 2 & Beginn der Therapie & erste Dosis & $70 \%$ der MED \\
\hline \multirow[t]{4}{*}{ Schritt 3} & $\begin{array}{l}\text { folgende Behandlung } \\
\text { drei- bis fünfmal pro } \\
\text { Woche }\end{array}$ & kein Erythem & $\begin{array}{l}\text { Steigerung um } \\
30 \%\end{array}$ \\
\hline & & minimales Erythem & $\begin{array}{l}\text { Steigerung um } \\
20 \% \text { (15\% nach } \\
2 \text { Behandlungen) }\end{array}$ \\
\hline & & $\begin{array}{l}\text { persistierendes } \\
\text { asymptomatisches } \\
\text { Erythem }\end{array}$ & keine Steigerung \\
\hline & & $\begin{array}{l}\text { schmerzhaftes } \\
\text { Erythem mit oder } \\
\text { ohne Ödem oder } \\
\text { Blasenbildung }\end{array}$ & $\begin{array}{l}\text { keine Bestrahlung } \\
\text { bis zum Abklingen } \\
\text { der Symptome }\end{array}$ \\
\hline Schritt 4 & $\begin{array}{l}\text { Wiederaufnahme } \\
\text { der Therapie }\end{array}$ & $\begin{array}{l}\text { nach Abklingen der } \\
\text { Symptome }\end{array}$ & $\begin{array}{l}\text { Reduktion der } \\
\text { letzten Dosis um } \\
50 \% \text {, weiter Steige- } \\
\text { rungen um } 10 \%\end{array}$ \\
\hline
\end{tabular}


Beim Wechsel zwischen verschiedenen Strahlertypen, z. B. zwischen Breitspektrum- und Schmalspektrumgeräten, sind erhebliche Dosiskorrekturen, am besten auf der Basis einer neuen Bestimmung der MED, notwendig.

Nach Erreichen der Erscheinungsfreiheit ist eine langfristig angelegte Erhaltungstherapie nicht angezeigt. Beim Auftreten von Rezidiven wird die Bestrahlungsfrequenz bis zum Erreichen der Erscheinungsfreiheit gesteigert. Dabei kann auch die UV-Dosis gesteigert werden. Zur Vermeidung hoher Kumulativdosen sollten jedoch Kombinationsbehandlungen erfolgen und es kann ein Wechsel zu einer anderen Therapieform sinnvoll sein.

\section{Excimer-Laser}

Eine neue Entwicklung zur Behandlung umschriebener Psoriasisherde ist die Anwendung eines Excimer-Lasers, der monochromatisch eine Strahlung von $308 \mathrm{~nm}$ erzeugt. Es existieren unterschiedliche Behandlungsprotokolle, wobei entweder niedrige Einzeldosen von 1 bis 3 MED mehrfach (ca. zehnmal) zur Anwendung kommen oder hohe Einzeldosen ( 8 bis 16 MED) nur einmalig angewandt werden. Ansprechraten schwanken zwischen 50 und $95 \%$ und die Remissiondauer beträgt drei bis fünf Monate [14-16]. Das Nebenwirkungsspektrum umfasst Erytheme und Blasenbildung in fast $50 \%$ der Fälle sowie Pigmentverschiebungen und Erosionen in 37 bzw. 25\% [14]. Der Vorteil der Behandlung liegt in der Schonung der nicht betroffenen gesunden Haut und in den möglicherweise längeren Remissionszeiten im Vergleich zur konventionellen Behandlung. Nachteile sind ein erheblicher technischer und mit hohen Kosten verbundener Aufwand, Nebenwirkungen und das bisher ungeklärte Risikoprofil. Weitere Studien sind erforderlich, um den Stellenwert der Excimer-Lasertherapie zu definieren.

\section{PUVA-Therapie}

Grundsätzlich stehen die systemische orale und die topische Photochemotherapie zur Verfügung. Die topischen Anwendungen gliedern sich in Vollbäder, Teilbäder und Cremeanwendungen. Nach wie vor umstritten ist die Nutzen-Risiko-Relation zwischen systemischer Photochemotherapie und Bade-PUVA-Behandlung. Die Bade-Photochemotherapie wurde bereits 1976 in Skandinavien eingeführt [17], und sie stellt dort bis heute den Standard dar. Seit Mitte der 80er-Jahre wurde die Behandlung auch in Mitteleuropa und Nordamerika zunehmend propagiert. Es liegt eine Reihe von Studien vor, die die Wirksamkeit der Bade-PUVA-Behandlung der Psoriasis darstellen und größtenteils mit einer systemischen PUVA-Therapie gleichsetzen [18-21]. Allerdings vermerken die Kostenträger kritisch, dass größere prospektive und randomisiert kontrollierte Studien bisher fehlen. Die Auswertung einer derzeit laufenden deutschen multizentrischen Studie wird mit Spannung erwartet.

Es darf nicht übersehen werden, dass auch die Bade-PUVA-Therapie ein potenzielles Karzinomrisiko beinhaltet. Bei der systemischen PUVA-Behandlung ist mittlerweile bei einer größeren Anzahl von Behandlungen (ca. 200) der Anstieg des Risikos für das Auftreten von spinozellulären Karzinomen der Haut gesi- chert [22,23]. Einhellig konnten jedoch bisherige epidemiologische Studien ein vergleichbares Risiko der Bade-PUVA-Behandlung nicht aufzeigen [24]. Hier vermögen methodische Ursachen und die vergleichsweise geringeren Fallzahlen verantwortlich sein. In jedem Fall zeigt die bisherige klinische Erfahrung, dass ein drastisch erhöhtes Karzinomrisiko nicht vorliegen kann.

\section{Praktisches Vorgehen bei Photochemotherapie}

Die unterschiedlichen Verfahren der Photochemotherapie und die dafür jeweils gebräuchlichen Dosierungen und Konzentrationen des Photosensibilisators sind in der Tab. $\mathbf{4}$ zusammengefasst.

Tab. 4 Unterschiedliche Verfahren der Photochemotherapie

\begin{tabular}{lll}
\hline Verfahren & Photosensibilisator & Dosis oder Konzentration \\
\hline \multirow{2}{*}{ PUVA oral } & 8-Methoxypsoralen (8-MOP) & $0,6 \mathrm{mg} / \mathrm{kg} \mathrm{KG}$ \\
& 5-Methoxypsoralen (5-MOP) & $1,2 \mathrm{mg} / \mathrm{kg} \mathrm{KG}$ \\
PUVA-Bad & 8-Methoxypsoralen (8-MOP) & $0,5-1,0 \mathrm{mg} / \mathrm{l}$ \\
& Trimethylpsoralen (TMP) & $0,33 \mathrm{mg} / \mathrm{l}$ \\
\multirow{2}{*}{ Creme-PUVA } & 8-Methoxypsoralen (8-MOP) & $\begin{array}{l}0,005-0.0006 \% \\
\text { Grundlagen: Unguentum } \\
\end{array}$ \\
& & $\begin{array}{l}\text { Cordes mit 30\% } \mathrm{H}_{2} \mathrm{O} \text { (DAB 9) } \\
\text { oder Cold Cream Nature }{ }^{\mathrm{TM}}\end{array}$ \\
\hline
\end{tabular}

* Im oberen Konzentrationsbereich ist bei großflächiger Anwendung mit systemischen Effekten durch Resorption zu rechnen.

Wegen der potenziellen akuten und chronischen Risiken einer Photochemotherapie ist die Indikation streng zu stellen. Auch stellt sie besondere Anforderungen an die Mitwirkung des Patienten.

Die folgenden Kontraindikationen sind zu beachten:

- Eine Kombinationsbehandlung mit Cyclosporin A ist zu vermeiden.

- Besondere Vorsicht ist geboten bei Vorbehandlung mit Arsen oder Röntgenstrahlen, bei gleichzeitiger Einnahme photosensibilisierender Medikamente, bei ausgeprägter Leberschädigung - sie verzögert bei metabolischer Insuffizienz die Sensibilisierungsphase - und bei hohen kumulativen UV-A-Dosen (mehr als 150 bis 200 Einzelbestrahlungen). In Einzelfällen können diese Umstände eine Kontraindikation bedeuten.

- Bezüglich des Risikos einer Kombination mit systemischen Fumaraten existieren keine Erfahrungsberichte.

- Eine Kombination der Photochemotherapie mit Methotrexat ist möglich.

Bei der oralen PUVA-Behandlung mit 8-MOP wird der Photosensibilisator ein (Flüssigkapseln) bzw. zwei Stunden (Tabletten) vor der UV-A-Bestrahlung eingenommen. Es wird ein Serumspiegel von durchschnittlich 100 bis $250 \mathrm{ng} / \mathrm{ml}$ 8-MOP erreicht. Die Spiegel können inter- und intra-individuell stark schwanken.

Bei der Bade-PUVA-Behandlung werden die 8-MOP-Konzentrationen im Badewasser mithilfe einer alkoholischen Stammlösung hergestellt. Die Badedauer beträgt 15 Minuten, die Tempe- 
ratur des Wassers $37^{\circ} \mathrm{C}$. Im unmittelbaren Anschluss an die Volloder Teilbädern muss die UV-A-Bestrahlung erfolgen, da die Sensibilisierung der Haut rasch abnimmt und nach ein bis zwei Stunden kaum mehr nachweisbar ist.

Die 8-MOP-Creme soll nach dem Auftragen eine Stunde einwirken. Danach erfolgt unmittelbar die Bestrahlung.

Die Patienten sind auf den konsequenten Lichtschutz der Haut, und bei PUVA oral auch der Augen, während der Sensibilisierungsphase hinzuweisen. Sie beträgt nach oraler Einnahme von 8-MOP etwa acht bis zehn Stunden, nach der PUVA-Bad-Behandlung ein bis zwei Stunden und nach einer 8-MOP-Creme-Anwendung zwei bis drei Stunden.

Die UV-A-Erstdosis richtet sich nach der individuellen Empfindlichkeit für die phototoxische Reaktion, die durch die minimale phototoxische Dosis (MPD) charakterisiert ist. Dabei nimmt die Lichtempfindlichkeit mit dem Ausmaß der phototoxischen Reaktion von Hauttyp IV zu Hauttyp I zu. Anstatt der Festlegung der ersten Bestrahlungsdosis durch die Bestimmung der minimalen phototoxischen Dosis, kann auch ein standardisiertes Verfahren entsprechend dem Photohauttyp (Tab. 5) erfolgen.

Die Bestimmung der phototoxischen Dosis wird mit einem Strahler, der dieselbe spektrale Emission wie das Therapiegerät aufweist, vorgenommen. Auch wird der Photosensibilisator in der jeweiligen, für die Behandlung vorgesehenen Applikationsweise, sowohl im Hinblick auf Dosis wie Konzentration, verwendet. Für die systemische Anwendung und Bade-PUVA-Therapie sind in Tab. 6 übliche Testdosen, abhängig vom Hauttyp, zusammengefasst. Unterschiede bei anderen Dosierungen oder 8-MOPKonzentrationen im Badewasser sind zu berücksichtigen. Während der Testbestrahlung wird der übrige Körper vollständig abgedeckt. Die Ablesung der Testareale erfolgt bei oraler Anwen-

Tab. 5 Empfehlungen für die Anfangsdosis bei Photochemotherapie

\begin{tabular}{llll}
\hline Photohauttyp & $\begin{array}{l}\text { PUVA oral } \\
\text { (8-MOP) } \\
\text { J/cm }\end{array}$ & $\begin{array}{l}\text { PUVA oral } \\
\text { (5-MOP) } \\
\text { J/cm }\end{array}$ & $\begin{array}{l}\text { PUVA-Bad } \\
\mathbf{( 1 , 0 ~} \mathbf{m g} / \mathbf{l} \text { 8-MOP) } \\
\mathbf{J} / \mathrm{cm}^{2}\end{array}$ \\
\hline I & 0,3 & 0,4 & 0,2 \\
\hline II & 0,5 & 1,0 & 0,3 \\
III & 0,8 & 1,5 & 0,4 \\
\hline IV & 1,0 & 2,0 & 0,6 \\
\hline
\end{tabular}

Tab. 6 Dosisempfehlungen für die Bestimmung der minimalen phototoxischen Dosis (MPD)

\begin{tabular}{lllllllll}
\hline Verfahren & Hauttyp & \multicolumn{1}{c}{ UV-A-Dosis $\left(\mathbf{J} / \mathbf{c m}^{2}\right)$} \\
\hline PUVA oral, 8-MOP & I-IV & 0,5 & 1 & 2 & 3 & 4 & 5 \\
\hline PUVA oral, 5-MOP & I-IV & 1 & 2 & 4 & 6 & 8 & 10 \\
PUVA-Bad (1 mg/l 8-MOP) & I, II & 0,25 & 0,5 & 1,0 & 1,5 & 2,0 & 2,5 \\
& III, IV & 0,5 & 1 & 2 & 3 & 4 & 5 \\
\hline
\end{tabular}

dung 72 bis 96 Stunden, bei Bade-PUVA 96 bis 120 Stunden nach Bestrahlung. Als Testareal empfiehlt sich eine lichtempfindliche, nicht-sonnengewöhnte Körperstelle (z.B. das Gesäß). Die Bestimmungen der MPD bei PUVA oral sind wegen der nicht gleichbleibenden Resorption des Photosensibilisators nicht absolut verlässlich. Die Werte sind jedoch bei der PUVA-Bad-Methode reproduzierbar und zur Steuerung der Behandlung hilfreich. Bei Creme-PUVA können Auftragsmenge und Resorption schwanken und zu variierenden Ergebnissen führen. Bei der Photochemotherapie palmoplantarer Dermatosen ist die Bestimmung der MPD nicht sinnvoll.

Ziel der intensiven initialen Behandlungsphase ist es, klinische Erscheinungsfreiheit zu erreichen. Die Bestrahlungen erfolgen in der Regel viermal pro Woche, mindestens aber zweimal wöchentlich. Dosissteigerungen sollten nicht häufiger als zweimal pro Woche und mindestens im Abstand von 72 Stunden vorgenommen werden [25]. Es existiert kein fixes Schema für Dosissteigerungen, die wesentlichen Parameter sollten das Ansprechen der behandelten Hauterkrankungen und die individuelle Empfindlichkeit gegenüber der Photochemotherapie sein. Ein gerade noch erkennbares Erythem wird als klinischer Indikator für eine ausreichende UV-A-Dosierung angesehen.

Wegen des höheren Grades der Sensibilisierung sind die Bestrahlungsdosen bei der Bade-PUVA-Behandlung generell geringer. Auch gilt es dabei darauf zu achten, dass die MPD während der ersten Therapiewoche vom Ausgangswert um bis zu 50\% absinken kann, später aber wieder ansteigt. Der Grund dafür ist nicht genau bekannt, es wird vermutet, dass Psoralen-Monoaddukte in der DNS persistieren und bei den darauf folgenden Bestrahlungen in phototoxisch wirksame Biaddukte umgewandelt werden. Generell empfiehlt es sich, bei der Bade-PUVA-Behandlung nur einmal wöchentlich die Dosis zu erhöhen oder diese zumindest in der ersten Woche konstant zu halten.

Ist der Patient klinisch erscheinungsfrei, so wird die Therapie bei gleich bleibender UV-A-Dosis, entsprechend der letzten effektiven Dosis der Initialtherapie, als Intervallbehandlung fortgesetzt. Dabei erhält der Patient einen Monat lang zwei Behandlungen pro Woche und einen weiteren Monat lang eine Behandlung pro Woche. Bleibt weiterhin Erscheinungsfreiheit bestehen, so wird die Therapie beendet. Wie auch bei der UV-B-Therapie ist der Nutzen dieser Intervalltherapie nicht hinreichend dokumentiert. Von einer lang andauernden Erhaltungstherapie ist abzusehen. Aufgrund internationaler Empfehlungen soll zur Minimierung des Langzeitrisikos die Anzahl der PUVA-Behandlungen 150 bis 200 nicht übersteigen. Bei Rezidiven kann während der Intervallphase die Bestrahlungsfrequenz wieder vorübergehend bis zur Erscheinungsfreiheit gesteigert werden. Bei Therapieresistenz sind Kombinationsbehandlungen oder andere Therapieschemata zu wählen.

Die in der Tab. 7 dargestellte Vorgehensweise hat sich in der Praxis bewährt. 
Tab. 7 Dosisschema für die Photochemotherapie

\begin{tabular}{|c|c|c|c|c|}
\hline Verfahren & & & PUVA oral & PUVA-Bad \\
\hline Schritt 1 & $\begin{array}{l}\text { Bestimmung der minimalen } \\
\text { phototoxischen Dosis (MPD) }\end{array}$ & & Ablesung nach $72-96 \mathrm{~h}$ & Ablesung nach $96-120 \mathrm{~h}$ \\
\hline Schritt 2 & Behandlungsbeginn & erste Behandlungsdosis & $\begin{array}{l}50-70 \% \text { der MPD oder Standard- } \\
\text { schema nach Hauttyp }\end{array}$ & $\begin{array}{l}30 \% \text { der MPD oder Standardschema } \\
\text { nach Hauttyp }\end{array}$ \\
\hline \multirow[t]{4}{*}{ Schritt 3} & $\begin{array}{l}\text { Fortsetzung der Behandlung } \\
\text { zwei- bis viermal pro Woche }\end{array}$ & kein Erythem, gutes Ansprechen & $\begin{array}{l}\text { Steigerung um } 30 \% \\
\text { max. zweimal wöchentlich }\end{array}$ & $\begin{array}{l}\text { Steigerung um } 30 \% \\
\text { max. zweimal wöchentlich }\end{array}$ \\
\hline & & minimales Erythem & keine Steigerung & keine Steigerung \\
\hline & & $\begin{array}{l}\text { persistierendes asymptomatisches } \\
\text { Erythem }\end{array}$ & keine Steigerung & keine Steigerung \\
\hline & & $\begin{array}{l}\text { schmerzhaftes Erythem mit oder } \\
\text { ohne Ödem oder Blasenbildung }\end{array}$ & keine Behandlung bis zum Abklingen & keine Behandlung bis zum Abklingen \\
\hline Schritt 4 & $\begin{array}{l}\text { Wiederaufnahme der } \\
\text { Behandlung }\end{array}$ & nach Abklingen der Symptome & $\begin{array}{l}\text { Reduktion der letzten Dosis um } 50 \% \text {, } \\
\text { weitere Steigerungen um } 10 \%\end{array}$ & $\begin{array}{l}\text { Reduktion der letzten Dosis um } 50 \% \text {, } \\
\text { weitere Steigerungen um 10\% }\end{array}$ \\
\hline
\end{tabular}

\section{Wirkungsmechanismen der Photo(chemo)therapie}

Das alte Konzept, die UV-Bestrahlung würde zu einer Bremsung der beschleunigten mitotischen Aktivität der Keratinozyten bei Psoriasis führen und diese damit günstig beeinflussen, kann nicht weiter aufrechterhalten werden. Als die wesentlichen Wirkungsmechanismen werden derzeit immunmodulatorische Einflüsse mit Überwiegen einer Immunsuppression sowie die Induktion einer Apoptose angenommen. Immunsuppressiv wirken die Depletion und der Funktionsverlust von antigenpräsentierenden Langhans-Zellen der Epidermis und dendritischen Zellen der Dermis [26]. Ebenso wird die Expression von Adhäsionsmolekülen (ICAM-1) an den Keratinozyten verringert und durch Induktion von immunsuppressiv wirkendem Interleukin 10 Interferon- $\gamma$ reduziert [27]. Apoptose entsteht nicht nur in den Keratinozyten der Epidermis, sondern insbesondere auch in den die Epidermis infiltrierenden T-Zellen. Die Reduktion der T-Zellen kann die Verminderung des entzündlichen Infiltrats bei Psoriasis, anderen entzündlichen Erkrankungen und insbesondere bei der Mycosis fungoides erklären [28].

\section{Risiken und Nebenwirkungen der Photo(chemo)therapie}

Die häufigsten akuten Nebenwirkungen umfassen sonnenbrandähnliche Erytheme nach UV-B-Bestrahlung sowie überschießende phototoxische Reaktionen bei Überdosierung der Bestrahlung im Rahmen der Photochemotherapie. Bei fehlendem Augenschutz können akute Konjunktivitis und Keratitis, chronisch, bei PUVA-Behandlung, eine Katarakt auftreten. Werden unbeabsichtigt phototoxische Substanzen oder Medikamente äußerlich bzw. systemisch zugeführt, so können während der Photochemotherapie überschießende phototoxische Hautreaktionen entstehen.

Spezifisch für die Photochemotherapie ist das gelegentliche Auftreten des PUVA-Juckreizes, der therapeutisch kaum beeinflussbar ist und manchmal zur Aufgabe der Therapie zwingt. Selten treten unter der PUVA-Behandlung bei starker mechanischer Belastung akrale Blasen infolge einer Lockerung der dermoepidermalen Grenzzone auf. Die mögliche systemische Toxizität durch
Tab. 8 Risiken und Nebenwirkungen der Photo(chemo)therapie

\begin{tabular}{|c|c|c|}
\hline & $U V-B$ & PUVA \\
\hline $\begin{array}{l}\text { Sonnenbrand oder phototoxische Reaktion bei } \\
\text { Überdosierung }\end{array}$ & ++ & ++ \\
\hline $\begin{array}{l}\text { Photoxische Reaktion durch unbeabsichtigte } \\
\text { Zufuhr eines Photosensibilisators }\end{array}$ & \pm & ++ \\
\hline $\begin{array}{l}\text { Konjunktivitis und Keratitis } \\
\text { (bei fehlendem Augenschutz) }\end{array}$ & ++ & ++ \\
\hline Provokation von Photodermatosen (PLD) & + & \pm \\
\hline UV-Lentigines & + & ++ \\
\hline Lichtalterung der Haut & ++ & ++ \\
\hline Präkanzerosen und spinozelluläres Karzinom & + & ++ \\
\hline Melanome & $?$ & $?$ \\
\hline
\end{tabular}

$(++)$ hohes Risiko; (+) mässiges Risiko; ( \pm ) geringes Risiko; (?) prinzipiell möglich, aber es existieren keine Daten.

Photochemotherapie, verursacht durch den Photosensibilisator, umfasst, Übelkeit nach der Tabletteneinnahme und gelegentlich Kopfschmerzen oder Schwindelgefühl.

Als chronische Schäden einer Photo(chemo)therapie treten UVinduzierte Lentigines, eine lichtinduzierte Hautalterung und insbesondere die Entwicklung von Präkanzerosen und Hautkrebs, auf. Statistisch gesichert ist die Entwicklung von spinozellulären Karzinomen nach systemischer PUVA-Behandlung mit hohen kumulativen UV-A-Dosen [22,23]. Nach PUVA-Bad-Behandlung wurde dies bislang nicht beobachtet [24]. Dies kann auf methodischen oder statistischen Mängeln der vorliegenden Untersuchungen beruhen. Bis zur Klärung dieser Frage ist daher auch für die PUVA-Therapie eine strenge Indikationsstellung zu fordern. In einer amerikanischen Studie wurde das vermehrte Auftreten von Präkanzerosen und Karzinomen des männlichen Genitale unter PUVA-Therapie gefunden [29]. Eine Bestätigung dieser Beobachtung steht jedoch aus. Basalzellkarzinome werden wahrscheinlich nur in geringem Maß durch die Photo(chemo)therapie induziert. Umstritten ist die Induktion von Melanomen durch PUVA [30]. Eine Zusammenfassung akuter und chronischer Nebenwirkungen der Photo(chemo)therapie gibt die Tab. 8. 
Die allgemeinen Richtlinien für eine sichere und wirksame Durchführung einer Photo(chemo)therapie sind die Folgenden:

- Beachtung der Indikationen und Kontraindikationen. Absolute Kontraindikationen sind Gendefekte mit einer erhöhten Lichtempfindlichkeit oder einem erhöhten Hautkrebsrisiko wie zum Beispiel Xeroderma pigmentosum, Cockayne-Syndrom und Bloom-Syndrom. Besondere Vorsichtsmaßnahmen wie strenge Indikationsstellung, engmaschige Kontrollen und modifizierte Behandlungsschemata erfordern Patienten mit Krampfleiden, mit der unvermeidbaren Einnahme von photosensibilisierenden Medikamenten, mit malignen Hauttumoren in der Vorgeschichte, mit erhöhter Lichtempfindlichkeit und Patienten mit atypischen, (dysplastischen) Nävuszellnävi.

- Der Patient muss über Therapieablauf, Nebenwirkungen und mögliche Langzeitrisiken aufgeklärt werden und bei einer Photochemotherapie sein schriftliches Einverständnis geben.

- Die applizierten UV-Dosen sind in exakten strahlungsphysikalischen Einheiten $\left(\mathrm{J} / \mathrm{cm}^{2}, \mathrm{~mJ} / \mathrm{cm}^{2}\right)$ zu bestimmen und aufzuzeichnen. Nach Abschluss einer Bestrahlungsserie sind die kumulativen UV-Dosen wie auch die Anzahl der Bestrahlungen festzuhalten.

- Während der Therapie sind die Patienten in regelmäßigen Abständen (vorzugsweise wöchentlich) vom Arzt zu überwachen. Der Therapieerfolg, unerwünschte Wirkungen und Begleittherapien sind zu dokumentieren.

- Generell ist ein Augenschutz durch geeignete Brillen während der Bestrahlung erforderlich. Bei Nichtbefall sind auch die chronisch lichtexponierten Areale Gesicht, Nacken und Handrücken zu schützen. Dies gilt nicht bei der Behandlung einer Mycosis fungoides, bei der auch an klinisch erscheinungsfreier Haut einer Manifestation vorgebeugt werden soll.

- Eine Phototherapie bei Kindern ist grundsätzlich in jedem Lebensalter möglich, solange von dem Kind der Augenschutz toleriert wird und sein Verhalten so angepasst ist, dass es die Bestrahlungsprozedur erträgt. Allerdings ist eine strenge Indikationsstellung erforderlich; PUVA-Therapie ist nur für Ausnahmen vorgesehen. Vorzuziehen sind Breitband- und Schmalspektrum-UV-B sowie UV-A1 im niedrigen und mittleren Dosisbereich bis $50 \mathrm{~J} / \mathrm{cm}^{2}$. In jedem Fall erfordert die Behandlung eine individuelle Abwägung von Nutzen und Risiken. Die Dosierung muss vorsichtig sein und möglichst im Suberythembereich liegen. Niedrige kumulative Dosen sind anzustreben.

\section{Literatur}

${ }^{1}$ Green C, Lakshmipathi T, Johnson BE, Ferguson JA. Comparision of the efficacy and relapse rates of narrowband UVB (TL-01) monotherapy vs. etretinate (re-TL-01) vs. etretinate-PUVA (re-PUVA) in the treatment of psoriasis patients. Br J Dermatol 1992; 127: 5-9

${ }^{2}$ Housman TS, Rohrback JM, Fleischer AB Jr, Feldmann SR. Phototherapy utilization for psoriasis is declining in the United States. J Am Acad Dermatol 2002; 46: 557-559

${ }^{3}$ Subkommission „Physikalische Therapie in der Dermatologie“ der Qualitätssicherungskommission der DDG. Empfehlungen zur Phototherapie und Photochemotherapie. Akt Dermatol 2003; 29: 113-120

${ }^{4}$ Mahrle G. Phototherapie in Kombination mit Cignolin, Teer und Retinoiden. In: Braun-Falco O, Schill WB (Hrsg). Fortschritte der prakti-
${ }^{5}$ Marcil I, Stern RS. Squamous-cell cancer of the skin in patients given PUVA and ciclosporin: nested cohort crossover study. Lancet 2001; 358: $1042-1045$

${ }^{6}$ Paul CF, Ho VC, McGeown C, Christophers E, Schmidtmann B, Guillaume JC, Lamarque V, Dubertret L. Risk of malignancies in psoriasis patients treated with cyclosporine: a $5 \mathrm{y}$ cohort study. J Invest Dermatol 2003; 120: $211-216$

${ }^{7}$ Storbeck H, Hölzle E, Schürer N, Lehmann P, Plewig G. Narrow-band UVB $(311 \mathrm{~nm})$ versus conventional broad-band UVB with and without dithranol in phototherapy for psoriasis. J Am Acad Dermatol 1993; 28 : 227-231

${ }^{8}$ Hofer A, Fink-Puches R, Kerl H, Wolf P. Comparison of phototherapy with near vs. far erythemogenic doses of narrow-band ultraviolet $B$ in patients with psoriasis. Br J Dermatol 1998; 138: $96-100$

${ }^{9}$ Dawe RS, Wainwright NJ, Cameron H, Ferguson J. Narros-band (TL-01) ultraviolet $B$ phototherapy for chronic plaque psoriasis: three times or five times weekly treatment? Br J Dermatol 1998; 138: 833-839

${ }^{10}$ Cameron H, Dawe RS, Yule S, Murphy J, Ibbotson SH, Ferguson J. A randomized, observer-blinded trial of twice vs. three times weekly narrowband ultraviolett $\mathrm{B}$ phototherapy for chronic plaque psoriasis. $\mathrm{Br} \mathrm{J}$ Dermatol 2002; 147: 973-978

${ }^{11}$ Walters IB, Burack LH, Coven TR, Gilleaudeau P, Krueger JG. Suberythemogenic narrow-band UVB is markedly more effective than conventional UVB treatment of psoriasis vulgaris. J Am Acad Dermatol 1999; 40: $893-900$

${ }^{12}$ Markham T, Rogers S, Collins P. Narrowband UV-B (TL-01) phototherapy vs oral 8-methoxypsoralen psoralen-UVA for the treatment of chronic plaque psoriasis. Arch Dermatol 2003; 139: 325-328

${ }^{13}$ Tanew A, Radakovic-Fijan S, Schemper M, Hoenigsmann H. Narrowband UV-B phototherapy vs photochemotherapy in the treatment of chronic plaque-type psoriasis: a paired comparison study. Arch Dermatol 1999; 135: 519-524

14 Feldman SR, Mellen BG, Housman TS, Fitzpatrick RE, Geronemus RG, Friedman PM, Vasily DB, Morison WL. Eficacy of the 308-nm excimer laser for treatment of psoriasis: results of a multicenter study. J Am Acad Dermatol 2002; 46: 900-906

${ }^{15}$ Trehan M, Taylor CR. High-dose 308-nm excimer laser for the treatment of psoriasis. J Am Acad Dermatol 2002; 46: 732-737

${ }^{16}$ Trehan M, Taylor CR. Medium-dose 308-nm excimer laser for the treatment of psoriasis. J Am Acad Dermatol 2002; 47: 701 - 708

${ }^{17}$ Fisher T, Alsins J. Treatment of psoriasis with trioxalen baths and dysprosium lamps. Acta Derm Venereol 1974; 56: 383-390

18 Calzavara-Pinton PG, Ortel B, Hönigsmann H et al. Safety and effectiveness of an aggressive and individualized bath-PUVA regimen in the treatment of psoriasis. Dermatology 1994; 189: 256-259

${ }^{19}$ Collins P, Rogers S. Bath-water compared with oral delivery of 8-methoxypsoralen PUVA therapy for chronic plaque psoriasis. Br J Dermatol 1992; 127: $392-395$

${ }^{20}$ Lowe NJ, Weingarten D, Bourget T, Moy LS. PUVA therapy for psoriasis; comparison of oral and bath-water delivery of 8-methoxypsoralen. J Am Acad Dermatol 1986; 14: $754-760$

${ }^{21}$ Turjanmaa K, Salo H, Reunala T. Comparison of trioxsalen bath and oral methoxsalen PUVA in psoriasis. Acta Derm Venereol (Stockh) 1985; 65: 86-88

${ }^{22}$ Henseler T, Christophers E, Hönigsmann H, Wolff K. Skin tumors in the European PUVA study: eight year follow-up of 1643 patients treated with PUVA for psoriasis. J Am Acad Dermatol 1987; 16: 108 - 116

${ }^{23}$ Stern RS, Nichols KT, Vakeva LH. Malignant melanoma in patients treated for psoriasis with methoxsalen (psoralen) and ultraviolet A radiation (PUVA). N Engl J Med 1997; 336: 1041 - 1045

${ }^{24}$ Hannuksela-Svahn A, Sigurgeirsson B, Pukkala E, Lindelöf B, Berne B, Hannuksela M, Poikolainen K, Karvonen J. Trioxsalen bath PUVA did not increase the risk of squamous cell skin carcinoma and cutaneous malignant melanoma in a joint analysis of 944 Swedish and Finnish patients with psoriasis. Br J Dermatol 1999; 141: 497-501

${ }^{25}$ Henseler T, Wolff K, Hönigsmann H, Christopers E. The European PUVA study (EPS) on oral 8-methoxypsoralen photochemotherapy of psoriasis. A cooperative study among 18 European centres. Lancet 1981; $1: 853-857$

${ }^{26}$ Grabbe J, Welker P, Humke S, Grewe M, Schöpf E, Henz BM, Krutmann J. Highdose UVA-1 therapy, but not UVA/UVB therapy, decreases IgE binding cells in lesional skin of patients with atopic eczema. J Invest Dermatol 1996; 107: 419-423 
${ }^{27}$ Grewe M, Gyufko K, Krutmann J. Interleukin-10 production by cultured human keratinocytes: regulation by ultraviolet $\mathrm{B}$ and ultraviolet $\mathrm{A} 1$ radiation. J Invest Dermatol 1995; 104: 3-6

${ }^{28}$ Yoo EK, Rook AH, Elenitas R, Gasparro FP, Vowels BR. Apoptosis induction by ultraviolet light $A$ and photochemotherapy in cutaneous T-cell lymphoma: Relevance to mechanism of therapeutic action. J Invest Dermatol 1996; 107: 235-242
${ }^{29}$ Stern RS. Genital tumors among men with psoriasis exposed to psoralens and ultraviolet A radiation (PUVA) and ultraviolet $B$ radiation. The photochemotherapy follow-up study. N Engl J Med 1990; 322: $1093-1097$

${ }^{30}$ Stern RS, Lunder EJ. Risk of squamous cell carcinoma and methoxsalen (psoralen) and UV-A radiation (PUVA). A meta-analysis. Arch Dermatol 1998; $134: 1582-1585$ 